\title{
Correção cirúrgica como tratamento de meningocele associada à craniosquise em bezerro: relato de caso
}

[Surgical correction as treatment of meningocele associated with cranioschisis in a calf: case report]

\section{"Relato de Caso/Case Report"}

\author{
Denise Batista Nogueira ${ }^{1}$, Rodolfo Monteiro Bastos ${ }^{1}$, Laysa Mayara Soares Brito Rocha ${ }^{1}$, \\ Ediane Freitas Rocha ${ }^{2}$, Pedro Isidro da Nóbrega Neto ${ }^{1}$, \\ Eldinê Gomes de Miranda Neto ${ }^{1}$, Tatiane Rodrigues da Silva ${ }^{1 *}$
}

\author{
${ }^{1}$ Unidade Acadêmica de Medicina Veterinária, Universidade Federal de Campina Grande (UFCG), Patos-PB, Brasil. \\ ${ }^{2}$ Programa de Pós-Graduação em Medicina Veterinária, Universidade Federal de Campina Grande (UFCG), Patos-PB, Brasil. \\ *Autor para correspondência/Corresponding author: E-mail: tatianerodrigues.vet@gmail.com
}

\begin{abstract}
Resumo
O objetivo desse trabalho é descrever um caso de meningocele associada à craniosquise em um bezerro SRD, de três dias de idade, que nasceu com aumento de volume na porção frontal da cabeça, sem manifestação de alterações neurológicas ou sensibilidade na região. O exame físico associado ao radiográfico confirmaram a anomalia como sendo craniosquise associada a meningocele, que foi reparada cirurgicamente. $\mathrm{O}$ animal foi submetido ao protocolo anestésico com xilazina a $2 \%(0,05 \mathrm{mg} / \mathrm{kg})$ via intramuscular, a indução e manutenção transcorreu com uso do anestésico geral isofluorano via inalatória e no bloqueio local utilizou-se lidocaína $2 \%$ sem vasoconstrictor. Foi drenado do aumento de volume aproximadamente 1,5 litros de líquor e realizada uma incisão elíptica contornando a bolsa, mantendo pele suficiente nas bordas para posterior sutura. $\mathrm{O}$ tamanho da falha na região frontal era de $7 \mathrm{~cm}$ de diâmetro e após drenagem foi realizada sutura das bordas das meninges em padrão simples contínuo e dermorrafia em padrão simples separado, utilizando fio nylon 0,35 e nylon 0,40 respectivamente. No pós-operatório foi utilizado antibioticoterapia, anti-inflamatórios, vitamina B1 e furosemida. A limpeza da ferida cirúrgica foi realizada diariamente com álcool iodado, spray cicatrizante e a retirada dos pontos ocorreu dez dias após o procedimento. Seguidos 39 dias o animal recebeu alta, apresentando-se saudável e sem deformidade ou recidivas do acúmulo de líquor. O tratamento cirúrgico foi eficaz e deve ser utilizado em casos semelhantes. Para redução do tempo quanto a recuperação dos pacientes, novas técnicas devem ser avaliadas, como a utilização de enxertos para correção da falha craniana em ruminantes.
\end{abstract}

Palavras-Chave: malformação; disrafia craniana; meninge; ruminantes.

\begin{abstract}
The objective of this work is to describe a case of meningocele associated with craniosquisis in a three dayold mixed breed calf that was born with increased volume in the frontal portion of the head, without neurological manifestation or sensitivity in the region. The physical examination associated with the radiographic exams confirmed the anomaly as craniosquisis associated with meningocele, which was surgically repaired. The animal was anesthetized with $2 \%$ xylazine $(0.05 \mathrm{mg} / \mathrm{kg})$ intramuscularly; induction and maintenance was carried out with isoflurane general anesthetic by inhalation, and in the local block, $2 \%$ lidocaine without vasoconstrictor was used. Approximately 1.5 liters of CSF was drained from the volume increase and an elliptical incision was made bypassing the pouch, maintaining sufficient skin at the edges for further suturing. The size of the fault in the frontal region was $7 \mathrm{~cm}$ in diameter and after drainage, the edges of the meninges were sutured in simple continuous pattern and the skin in a simple interrupted pattern. In the postoperative period antibiotic therapy, anti-inflammatories, vitamin B1, and furosemide were used. After 39 days, the animal was discharged, being healthy and without deformity or relapses of CSF accumulation. Surgical treatment was effective and should be used in similar cases. To reduce the time to recovery of patients, new techniques should be evaluated, such as the use of grafts to correct cranial failure in ruminants.
\end{abstract}

Keywords: malformation; cranial dysraphy; meningitis; ruminant. 


\section{Introdução}

As malformações congênitas ocorrem em um ou mais estágios do desenvolvimento fetal, surgindo como consequência de fatores intrínsecos (genéticos) ou extrínsecos (infecciosos, tóxicos e mecânicos) ou pela interação entre ambos. Além disso, muitas malformações ocorrem de forma esporádica sem uma causa conhecida (Leipold e Dennis, 1980).

No sistema nervoso central (SNC) essas alterações são anormalidades intrínsecas que ocorrem durante o processo de formação do tubo neural. O SNC dos animais domésticos é altamente susceptível aos agentes teratogênicos, principalmente pelo alto grau de diferenciação e complexidade do tecido nervoso (Zachary, 2013; Ecco et al., 2016).

A craniosquise é um tipo de disrafia (falha na ossificação), que ocorre na linha média do crânio, resultando em comunicação direta entre a meninge e a pele que recobre a região, caracterizando uma herniação da meninge repleta de líquido cefalorraquidiano (meningocele), onde usualmente apresenta projeção do tecido meningeal (Zachary, 2013).

Anormalidades congênitas do encéfalo podem ser causadas por alterações na morfogênese ou na histogênese do tecido nervoso, ou decorrerem de falhas no desenvolvimento de estruturas associadas (notocorda, somitos, mesênquima e crânio). Defeitos na formação do crânio (cranium bifidum) estão muitas vezes, associados a malformações congênitas do cérebro e/ou meninges. Tais defeitos do crânio estão usualmente no plano médio e quase sempre na calota craniana (epicrânio). Experimentos genéticos mostraram hereditariedade de caráter recessivo com penetrância variável (Leipold e Dennis, 1980; Moore e Persaud, 2008).

De uma forma geral, os animais acometidos com algum tipo de malformação possuem um prognóstico reservado a ruim e se tratando de craniosquise em ruminantes, são poucos os relatos de tratamento realizado com sucesso. O objetivo do presente trabalho é relatar a correção cirúrgica realizada como forma de tratamento em um bezerro com craniosquise e meningocele.

\section{Descrição do caso}

Em agosto de 2016 foi atendido no Hospital Veterinário da Universidade Federal de Campina Grande (HOVET-UFCG), Campus de Patos,
Paraíba, um bezerro, macho, sem padrão de raça definido, com três dias de idade e pesando $18 \mathrm{Kg}$, que segundo proprietário, apresentava um aumento de volume na região frontal da cabeça desde o seu nascimento, que ocorreu de forma eutócica.

Foi realizado exame físico geral e específico do sistema nervoso central além de exame radiográfico da região cranial da cabeça (projeções latero-lateral e dorso ventral), para elucidação do diagnóstico presuntivo e determinação do tratamento e prognóstico.

Ao exame físico geral e do sistema nervoso o paciente apresentava todos os parâmetros fisiológicos normais para um animal de sua espécie e idade, no entanto havia um aumento de volume na cabeça, região frontal, medindo aproximadamente $25 \mathrm{~cm}$ de diâmetro e de consistência amolecida (Figura 1A). Os achados radiográficos consistiram em uma massa de formato redondo, bem definida e de aspecto radiopaco na região frontal do crânio (Figura 1B).

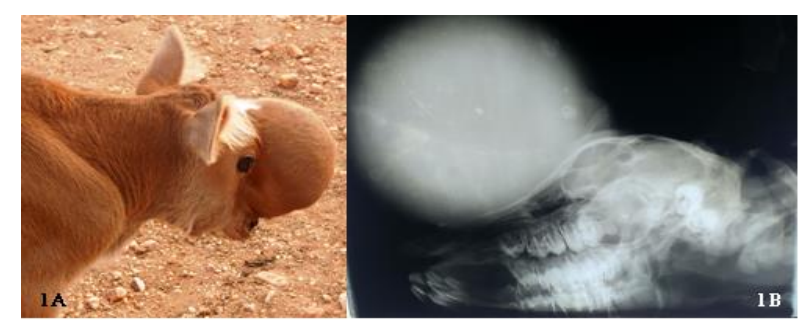

Figura 1. Bezerro com craniosquise associado a meningocele. A - Aumento de volume na região frontal medindo aproximadamente $25 \mathrm{~cm}$ de diâmetro, consistência amolecida e recoberto por pele íntegra. B - Ao exame radiográfico foi visualizado massa de formato arredondado, bem definida e de aspecto radiopaco.

De acordo com os achados clínicos e radiográficos o diagnóstico definitivo foi meningocele associada à craniosquise e buscando uma melhor qualidade de vida para o animal optouse pelo procedimento cirúrgico de drenagem do líquido cefalorraquidiano e sutura das meninges expostas e demorrafia.

O animal foi submetido ao protocolo anestésico com medicação pré-anestésica a base de xilazina $\left(\right.$ Xilazin $\left.^{\circledR}\right)$ a $2 \%$ na dose de $0,05 \mathrm{mg} / \mathrm{kg}$ por via intramuscular (IM) e então posicionado em decúbito esternal. A indução e manutenção transcorreu com o uso do anestésico geral isofluorano por via inalatória através de intubação traqueal com sonda $n^{\circ} 5$, tendo consumo total de 4 $\mathrm{mL}$ ao final da cirurgia. Para bloqueio local 
utilizou-se lidocaína $2 \%$ sem vasoconstrictor (Lidovet ${ }^{\circledR}$ ).

Foi drenado do aumento de volume, com auxílio de uma agulha hipodérmica (40x16) acoplada ao aspirador mecânico, aproximadamente 1,5 litro de líquor com aspecto sanguinolento (Figura 2A). Em seguida, foi realizada uma incisão elíptica contornando a bolsa, mantendo pele suficiente nas bordas para posterior sutura (Figura 2B). O tamanho da falha na região frontal era de aproximadamente $7 \mathrm{~cm}$ de diâmetro (Figura 2C), e após drenagem foi realizada sutura das bordas das meninges em padrão simples contínuo, interrompendo a cada $5 \mathrm{~cm}$ (nylon $0,35 \mathrm{~mm}$ ) e dermorrafia em padrão simples separado (nylon 0,40 mm) (Figura 2D). Uma hora após o término da cirurgia foi drenado cerca $500 \mathrm{~mL}$ de líquor remanescente, de aspecto sanguinolento. No pósoperatório foi utilizado antibioticoterapia com ceftiofur $4,4 \mathrm{mg} / \mathrm{kg}$ IM $\left(\right.$ Eficur $^{\circledR}$ ) por sete dias, como anti-inflamatórios flunixin meglumine 2,2 $\mathrm{mg} / \mathrm{kg}$ IV $\left(\right.$ Flumegan $^{\circledR}$ ) durante três dias, dexametasona $0,2 \mathrm{mg} / \mathrm{kg}$ IV (Cortvet $^{\circledR}$ ) por dois dias, 0,9 $\mathrm{mL}$ de vitamina B1 (tiamina) IM (Monovin $\mathrm{B} 1{ }^{\circledR}$ ) por 13 dias e furosemida $2,2 \mathrm{mg} / \mathrm{kg}$ IV (Semidin ${ }^{\circledR}$ ) durante seis dias. A limpeza da ferida cirúrgica foi realizada diariamente com álcool iodado, spray cicatrizante (Aerocid ${ }^{\circledR}$ ) e retirada da sutura dez dias após o procedimento.

Após a cirurgia foi notado que havia um aumento de volume gradativo na região cirúrgica e no $14^{\circ}$ dia pós-cirúrgico foi fixado um dreno e removido cerca de $750 \mathrm{~mL}$ de líquor da região frontal. Após 39 dias o animal recebeu alta, apresentando-se saudável e sem deformidade ou recidivas do acúmulo de líquor.

\section{Discussão}

Defeitos congênitos (DCs) são anormalidades na estrutura ou função de órgãos, presentes ao nascimento, acometendo-os de forma parcial ou completa (Rotta et al., 2008). Podem estar presentes em qualquer espécie animal, ocorrendo invariavelmente ao nascimento, tendo sua classificação designada de acordo com o órgão ou sistema primariamente acometido (Schild, 2007).

Dantas et al. (2010) realizaram um levantamento da ocorrência de malformações congênitas em ruminantes diagnosticadas entre 2000 e 2008 em municípios da Paraíba, Pernambuco e Rio Grande do Norte. Durante o período deste estudo foram recebidas 1.347 amostras, dos quais $47 \quad(3,48 \%)$ tiveram diagnóstico positivo para malformações congênitas. Em relação as causas, foram constatadas consumo de Mimosa tenuiflora e malformações esporádicas sem causa conhecida. Dentre o total de animais positivos, 14 eram bovinos, sendo oito imputados ao consumo de $M$. tenuiflora e seis malformações foram atribuídas a causas esporádicas; 21 eram ovinos, sendo 18 correspondentes a malformações por $M$. tenuiflora e três a causas esporádicas; 12 eram caprinos e destes nove causadas pela ingestão de $M$. tenuiflora e três devido malformações esporádicas. Em meio aos 47 animais diagnosticados com malformações, apenas em um bovino advindo do município Santa Terezinha-PE, foi observado presença de meningocele, o qual não teve causa definida e estava associado a coristoma pulmonar.

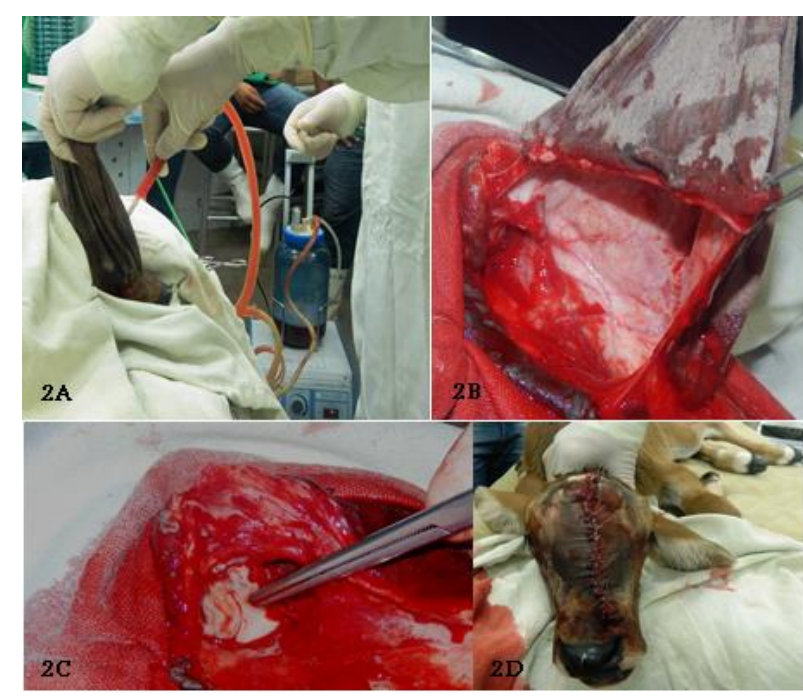

Figura 2 - Procedimento cirúrgico em bezerro com craniosquise associado a meningocele: $\mathbf{A}$ drenagem de aproximadamente 1,5 litro de líquor com aspecto sanguinolento do aumento de volume, com auxílio de uma agulha hipodérmica (40x16) acoplada ao aspirador mecânico. B - Incisão elíptica contornando a bolsa, mantendo pele suficiente nas bordas para posterior sutura. C - Falha na região frontal era de aproximadamente $7 \mathrm{~cm}$ de diâmetro. D - Dermorrafia em padrão simples separado (nylon 0,40 mm).

Souto et al. (2016) descreveram um caso de um bezerro com malformação que foi atendido no HOVET-UFCG no ano de 2012, no qual o diagnóstico anatomopatológico foi de meningocele, craniosquise e hipoplasia prosencefálica. Neste caso a abertura no crânio também ocorreu na linha média do osso frontal, assim como no presente relato, porém nesse primeiro caso, a meningocele assumiu uma postura 
pendular, devido ao acúmulo de líquor no seu interior e o quadro foi agravado devido à hipoplasia prosencefálica.

Macêdo et al. (2011) também realizaram uma revisão de casos de defeito congênito em bovinos na região central do Rio Grande do Sul, durante o período de 1964 a 2010. De um total de 7.132 bovinos necropsiados, 31 bezerros $(0,4 \%)$ apresentaram doenças congênitas, sendo que dentre estes somente quatro bovinos natimortos foram diagnosticados com craniosquise, sendo que um apresentava associado à hidrocefalia e três casos com meningocele.

Oliveira Filho et al. (2014) reportaram um caso de craniosquise com meningocele na região parietal de uma bezerra de dois dias de idade. Mediante esta contingência, optou-se pela correção cirúrgica através de cranioplastia utilizando um enxerto homólogo de cartilagem na tentativa de corrigir a falha óssea. Assim como o animal relatado no presente trabalho, esta bezerra não apresentava nenhuma anormalidade neurológica ao exame do sistema nervoso central e todos seus parâmetros estavam dentro da normalidade. Porém, um aumento de volume foi encontrado no osso parietal do crânio, com medidas de 13x10x6 cm. Foi revelada a presença de craniosquise associada à meningocele através de radiografias de contraste, as quais evidenciaram displasia óssea parietal e supraoccipital, ausência de ossos interparietais e acúmulo de líquido na massa. Para a correção cirúrgica utilizando enxerto, foi escolhida cartilagem auricular de bovinos adultos colhidas em matadouro e devidamente processadas, com o intuito de criar uma nova matriz óssea, mas após receber alta o bezerro foi a óbito.

A literatura traz alguns relatos de craniosquise e meningocele em bezerros, onde foi adotada a reparação cirúrgica como método de tratamento. Pode se destacar os relatos de Back et al. (1991) e Kohli e Nadaf (1998), o primeiro descreveu o caso de uma bezerra de dois meses e meio que apresentava uma protuberância localizada caudalmente a região intercornual. $\mathrm{O}$ segundo relata o caso de uma bezerra com cinco dias de idade, que demonstrava aumento de volume na região frontal, mais especificamente na região supraorbital. Nas duas ocorrências o tratamento cirúrgico através da cranioplastia foi bemsucedido.

Segundo Maranha et al. (2012), em humanos a craniosquise é uma malformação rara e de incidência indistinta, revisões sobre o tema mostram que de cada 10.000 nascimentos, 10 apresentam um defeito congênito, que geralmente está associado a anencefalia (malformação ou ausência do cérebro e/ ou da calota craniana).

\section{Conclusão}

O tratamento cirúrgico realizado foi eficaz e deve ser utilizado em casos semelhantes, no entanto, para redução do tempo quanto a recuperação dos pacientes, novas técnicas devem ser avaliadas, como a utilização da fixação de dreno durante a dermorrafia e a utilização de enxertos para correção da falha craniana em ruminantes.

\section{Conflito de interesse}

Os autores declaram não existir conflito de interesse.

\section{Referências}

Back, W.; Van Den Belt, A.J.M.; Lagerweij, E.; Van Overbeeke, J.J.; Van Der Velden, M.A Surgical repair of a cranial meningocele in a calf. Veterinary Record, 128(24): 569-71, 1991.

Dantas, A.F.M.; Riet-Correa, F.; Medeiros, R.M.T.; Galiza, G.J.N.; Pimentel, L.A.; Anjos, B.L.; Mota, R.A. Malformações congênitas em ruminantes no semiárido do Nordeste Brasileiro. Pesquisa Veterinária Brasileira, 30(10): 807-815, 2010.

Ecco, R.; Viott, A.M.; Graça, D.L.; Alessi, A.C. Sistema nervoso. In: Santos, R.L.; Alessi, A.C. Patologia veterinária. São Paulo: Roca, 2016. p.487-572.

Kohli, R.; Naddaf, H. Surgical treatment of cranial meningocele in Iranian calves. Veterinary Record, 142(6): 145, 1998.

Leipold H.W.; Dennis S.M. Congenital defects affecting bovine reproduction. In: Morrow D.A. Current therapy in theriogenology. (ed.) Philadelphia: W.B. Saunder Company, 1980. p. 410- 441.

Macêdo, J.T.S.A.; Lucena. R.B.; Giaretta, P.R.; Kommers, G.D.; Fighera, R.A.; Irigoyen, L.F.; Barros, C.S.L. Congenital defects in cattle from central Rio Grande do Sul, Brazil. Pesquisa Veterinária Brasileira, 31(4): 297306, 2011.

Maranha, L.A.; Augusto, L.P.; Zanine. S.C.; Araújo, J.C. Acrania e outras falhas na formação dos ossos do crânio: uma revisão de literatura. Jornal Brasileiro de Neurocirurgia, 23(3): 35-38, 2012. 
Moore, K.L.; Persaud, T.V.N.; Embriologia básica. $7^{\text {nd }}$ ed. Rio de Janeiro: Elsevier, 2008, $365 \mathrm{p}$.

Oliveira Filho, J.P.; Badial, P.R.; Oliveira, A.P.; Álvares, L.E.C.; Costa, J.L. O Cranioplasty for repair of cranioschisis associated with meningocele in a Jersey calf. Veterinária e Zootecnia, 21(3): 392-398, 2014.

Rotta, I.T.; Torres, M.B.A.M.; Motta, R.G. Diprosopia em bovino. Arquivo Brasileiro de Medicina Veterinária e Zootecnia, 60(2): 489-491, 2008.

Schild, A.L. Defeitos congênitos. In: Riet-Correa, F.; Schild, A. L.; Lemos, R. A. A.; Borges J.
R. J. Doenças de ruminantes e equídeos. $3^{\text {nd }}$ ed. Santa Maria: Pallotti, 2007. v.1. p.25-55.

Souto, E.P.F.; Oliveira Filho, A.F.; Carvalho, F.K.L.; Miranda Neto, E.G.; Dantas, A. F. M. Meningocele, craniosquise e hipoplasia prosencefálica em bezerro. Pesquisa Veterinária Brasileira, 36(0): 175-177, 2016.

Zachary, J.F. Sistema nervoso. In: McGavin, M.D.; Zachary, J.F. Bases da patologia veterinária. $5^{\text {nd }}$ ed. Rio do Janeiro: Elsevier, 2013. p.542591. 